\title{
Probability Boxes Theory Research Strategy in the Mechanical Equipment Healthy State Monitoring
}

\author{
Du $\mathrm{Yi}^{1}$ \\ City College \\ Kunming University of Science and Technology \\ Kunming, China \\ e-mail: duyi215@hotmail.com
}

\author{
Ding Jiaman ${ }^{2 *}$ \\ College of Information Engineering and Automation \\ Kunming University of Science and Technology \\ Kunming, China \\ e-mail: tjom2008@163.com
}

\author{
Liu $\mathrm{An}^{3}$ \\ Faculty of Mechanical and Electrical Engineering \\ Kunming University of Science and Technology \\ Kunming, China \\ e-mail: liuanmike@sina.com
}

\begin{abstract}
Known the health state of each mechanical part in advance is particularly important in mechanical equipment conditional monitoring. However, it is one of the most difficulties to be solved in mechanical equipment conditional monitoring. A new probability boxes theory research Strategy for the mechanical equipment healthy state monitoring was proposed to solve the above problem. Firstly, the probability box theory was introduced and the basic concepts and the relationship of probability box and Dampster Shafer structure were contracted. The feasibility was discussed then. Secondly, the relationship of probability box's compactness and data uncertainty was verified by a uncertainty Variables which obeyed by the exponential distribution. Finally, the probability box research strategy was proposed in details with a simulation rotating machinery fault test bed. The corresponding sensors were layout on the reasonable positions to monitor the various mechanical parts; the experimental data was Obtain by the corresponding sensor for the monitored objects. Different data of health state and fault state was obtained; the specification of probability box heath index was established. The results show that the strategy may be a promising healthy state monitoring method for Mechanical Equipment.
\end{abstract}

Keywords- Probability box theory; Research Strategy; Uncertainty; Healthy State Monitoring; Condition Monitoring

\section{INTRODUCTION}

The system uncertainties of mechanical equipment are different at various running stages. At initial stage, each parts or components are healthy and running smoothly. There is less uncertainties problem with the mechanical equipment system. With the increase of operation time, the uncertainties of the system increase gradually. Just like human being, the mechanical equipment enters into the sub-health state. With the further increase of operation time, some mechanical parts fail. These parts in fault state should be replaced to keep the system running.

Known the health state of each mechanical part in advance is particularly important in mechanical equipment conditional monitoring. However, it is one of the most difficulties to be solved in mechanical equipment conditional monitoring. The probability box (Referred as p-box) theory provides a new way to solve the above problem. The advantage of p-box theory is that it can express almost all types of uncertainty. The p-box can accurately express the quantity of uncertainty. A health state monitoring strategy of mechanical equipment based on the p-box theory was proposed.

The P-box theory comes from the probability distribution function under the condition of known probability distribution type. It was experienced the crossover study with fuzzy theory ${ }^{[1]}$, DS evidence theory ${ }^{[2]}$, based on the traditional probabilistic Boolean logic ${ }^{[3]}$, sparse sample Kolmogorov method ${ }^{[4]}$. It was integrated into the stochastic algorithm (such as Bayesian reasoning and evidence theory), but also into the artificial intelligent algorithm (such as set theory, neural network ${ }^{[5]}$, expert system, fuzzy theory $\left.{ }^{[6]}\right)$.The USA Sandia National Laboratory in cooperation with the America Energy Department released an uncertainty research report ${ }^{[7]}$ in 2003, which means that the probability box has entered a relatively mature stage in theory.

Its application has been the trend of diffusion in recent years. Its application areas include: the failure probability evaluation of fault system ${ }^{[8]}$, the uncertainty evaluation of vibration system dynamic responses ${ }^{[9]}$, The uncertainty of climate change ${ }^{[10]}$, Seawall risk modeling and reliability assessment ${ }^{[1-12]}$, Automobile gearbox reliability design ${ }^{[13]}$, finite element modeling and parameter optimization of rocket shell structure ${ }^{[14]}$, parameter uncertainty of damped oscillator $^{[15]}$, multi-parameters uncertainty mathematical modeling ${ }^{[16]}$, Mechanical reliability system architecture 
and evaluation ${ }^{[17]}$, The flood control evaluation of water conservancy system ${ }^{[18]}$, The error accumulated expression and evaluation of the measuring system ${ }^{[19]}$, the sea level estimation in the further considering climate change ${ }^{[20]}$, etc.

\section{PRoBABILITY BoXES AND DEMPSTER-SHAFE STRUCTURE}

There are two fundamentally distinct forms of uncertainty in the nature. The first is variability that arises from environmental stochasticity, inhomogeneity of materials, fluctuations in time, variation in space, or heterogeneity or other differences among components or individuals. Variability is sometimes called Type I uncertainty. Type I cannot be changed. The second kind of uncertainty is the incertitude that comes from scientific ignorance, measurement uncertainty, inobservability, censoring, or other lack of knowledge. This is sometimes called Type II uncertainty. Type II can be changed as the science development so most researches aim to Type II. Type I uncertainty is always represented by probability theory. Several disparate theories have been proposed to represent type II such as Intervals Analysis, Imprecise Probabilities. Dempster-Shafer Structure (DSS) was proposed by Shafe and the probability boxes (p-boxes) were proposed by Willianson. P-boxes and DSS can present both type I and type II uncertainties.

\section{A. Probability box}

Whether the probability distribution type is known or not, the upper and lower bounds are defined. The real cumulative probability will be limited in the area surrounded by the two bounds. The area is defined as pbox.

If $\bar{F}(x)$ and $\underline{F}(x)$ are increasing function of real numbers, which ranges are in $[0,1]$. For all $\mathrm{x} \in \mathfrak{R}$, there exists the relationship $\underline{F}(x) \leq \bar{F}(x)$. The interval $[\underline{F}(x), \bar{F}(x)]$ presents the upper and lower bounds of increasing function $\mathrm{F}(\mathrm{x})$ and satisfies the condition $\underline{F}(x) \leq F(x) \leq \bar{F}(x)$. The range $[\underline{F}(x), \bar{F}(x)]$ is called as a pair of function with an uncertainty distribution, which is called as a probability box.

For a random variable $\mathrm{X}$, assuming the probability distribution of $\mathrm{X}$ is unknown, $\underline{F}(x)$ is defined as the lower Cumulative distribution function (CDF) which presents the probability of $\mathrm{X} \leq \mathrm{x}$. Similarly, $\bar{F}(x)$ is defined as the upper CDF. The upper and lower bounds of the probability distribution function can be calculated by the probability measure $\underline{P}(x)$ :

$$
\begin{gathered}
\bar{F}_{X}(X)=1-\underline{P}(X>x) \\
\underline{F}_{X}(X)=\underline{P}(X \leq x)
\end{gathered}
$$

As shown in Fig .1, the left boundary $\bar{F}(x)$ is the upper boundary of the probability distribution, corresponding in the lower boundary of quantile $\mathrm{x}$. The right boundary $\underline{F}(x)$ is the lower boundary of the probability distribution, corresponding in the upper boundary of quantile $\mathrm{x}$.

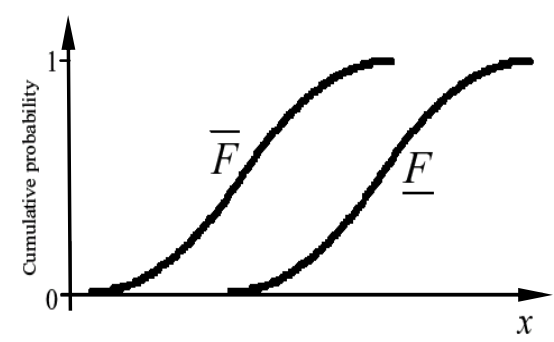

Figure 1. The Schematic diagram of a p-box

\section{B. Dempster Shafer Structure (DSS)}

A finite DSS on the real number field $\mathfrak{R}$ can be presented by its basic probability assignment function, which is a mapping relation:

$$
m: 2^{\Re} \rightarrow[0,1]
$$

$$
\begin{aligned}
& m(\Phi)=0, \\
& m\left(a_{i}\right)=p_{i}\left(a_{i} \subseteq \mathfrak{R}, i=1,2 \ldots n\right), \\
& m(D)=0\left(\mathrm{D} \neq a_{i}\right)
\end{aligned}
$$

Where:

$$
p_{i}>0 \text { and } \sum_{i=1}^{n} p_{i}=1
$$

The plausibility function (Pls): It's a sum of all the corresponding mass of a DSS which has intersection with the subset $b \subseteq \mathfrak{R}$, which can be presented by the following function:

$$
P l s(b)=\sum_{\substack{a \\ a \cap b \neq \varnothing}} m(a)=\sum_{\substack{i \\ a_{i} \cap b \neq \varnothing}} m\left(a_{i}\right)
$$

The belief function (Bel): It's a sum of all the corresponding mass of a DSS which is the subset of interval $b \subseteq \mathfrak{R}$, which can be presented by the following function:

$$
\operatorname{Bel}(b)=\sum_{\substack{a \\ a \subseteq b}} m(a)=\sum_{\substack{i \\ a_{i} \subseteq b}} m\left(a_{i}\right)
$$

A DSS is composed by the sets which including an interval and its corresponding mass. The form is as follows $\left\{\left(\left[\mathrm{x}_{1}, \mathrm{y}_{1}\right], \mathrm{m}_{1}\right),\left(\left[\mathrm{x}_{2}, \mathrm{y}_{2}\right]\right.\right.$, $\left.\left.\mathrm{m}_{2}\right), \ldots,\left(\left[\mathrm{x}_{\mathrm{n}}, \mathrm{y}_{\mathrm{n}}\right], \mathrm{m}_{\mathrm{n}}\right)\right\}$ and satisfying the following conditions:

$$
x_{i}<y_{i}, \quad \sum m_{i}=1, \quad y_{i} \neq y_{j}
$$




\section{The Relationship of P-box and DSS}

The lower bound of a p-box can be achieved by the sum of all the corresponding lower bounds of a DSS and the upper bound of a p-box can be achieved by the sum of all upper bounds of a DSS. If a DSS can be presented by $\left\{\left(\left[\mathrm{x}_{1}\right.\right.\right.$, $\left.\left.\left.\mathrm{y}_{1}\right], \mathrm{m}_{1}\right),\left(\left[\mathrm{x}_{2}, \mathrm{y}_{2}\right], \mathrm{m}_{2}\right), \ldots,\left(\left[\mathrm{x}_{\mathrm{n}}, \mathrm{y}_{\mathrm{n}}\right], \mathrm{m}_{\mathrm{n}}\right)\right\}$, the lower bound (Bel) function and the upper bound function of the corresponding p-box transformed from the DSS can be expressed by:

$$
\begin{aligned}
& \underline{F}(z)=\operatorname{Bel}(z)=\sum_{y_{i}<z} m_{i} \\
& \bar{F}(z)=\operatorname{Pls}(z)=\sum_{x_{i}<z} m_{i}
\end{aligned}
$$

The transformation process from a DSS to a p-box is not all information reserving. Some information in details will be lost. The p-box can also be transformed into a DSS by standard discretization.

A p-box can be composed of different DSS. The mass of the DSS's interval can be the same or not. In order to calculate conveniently, a p-box is always broken down into a DSS with the same mass. This process is also known as the discretization of a p-box as shown on Fig.2.

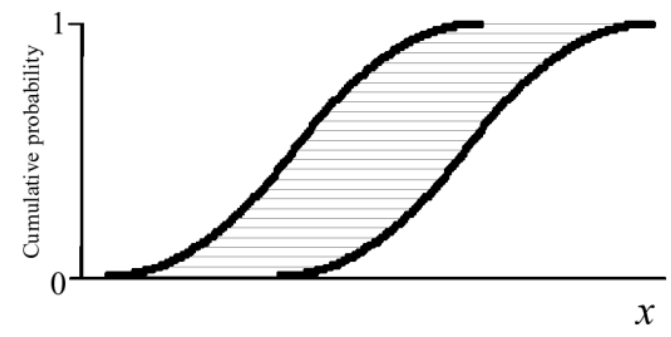

Figure 2. the Equiprobable discretization of a p-box

\section{THE RESEARCH STRATEGY OF PROBABILITY BOXES THEORY}

A. The relationship between the p-box compactness and data uncertainty

The p-box's compactness of mechanical part is the best in health state. With the extension of running time, the pbox's compactness is becoming worse. In order to illustrate the relationship between the p-box compactness and data uncertainty, an instance of p-box was proposed. An uncertainty variable $\mathrm{x}$ is assumed to be obeyed the exponential distribution. It rate parameter $(\lambda)$ 's fluctuation range is [2, 3], [1.5, 3.5], [1, 4], as shown in Fig .3.

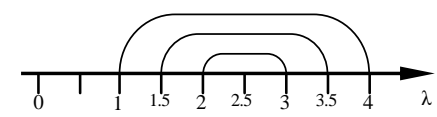

Figure 3. The flucturation range of rate parameter $(\lambda)$
The p-boxes results are shown in Fig .4, from which several conclusions can be analyzed as following: the smaller the fluctuation ranges become, the more compact the p-box express. With the parameters' fluctuation range increase, the compactness of p-box become worse, which means the uncertainties contained by $\mathrm{p}$-box are increasing.

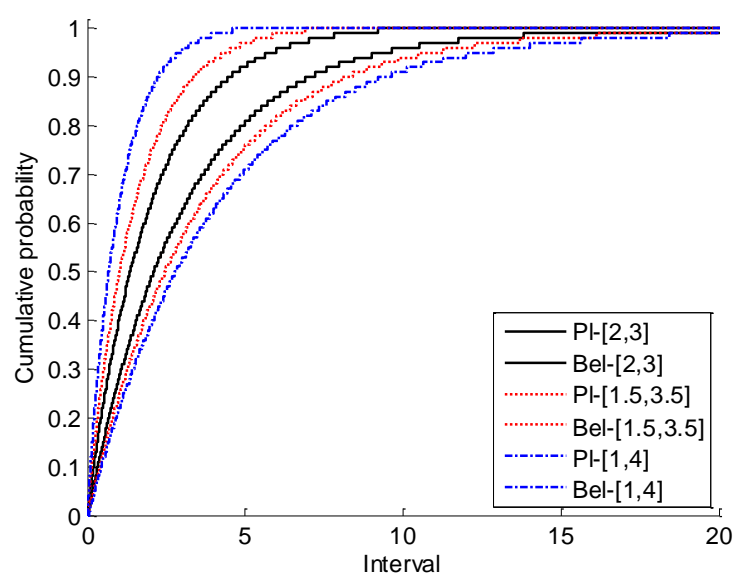

Figure 4. The p-boxes result of different compactness

\section{B. The research strategy for mechencial equipment healthy state monitoring}

The simulation rotating machinery fault test bed QPZZ-II was used to illustrate the strategy. Signal acquisition was used by the United States NI PXI-1042Q high performance acoustic vibration test system. The software was used by LabVIEW data acquisition software. Sensors were used by the United States PCB M603C01 ICP acceleration sensors. The experimental apparatus was shown in Fig .5.

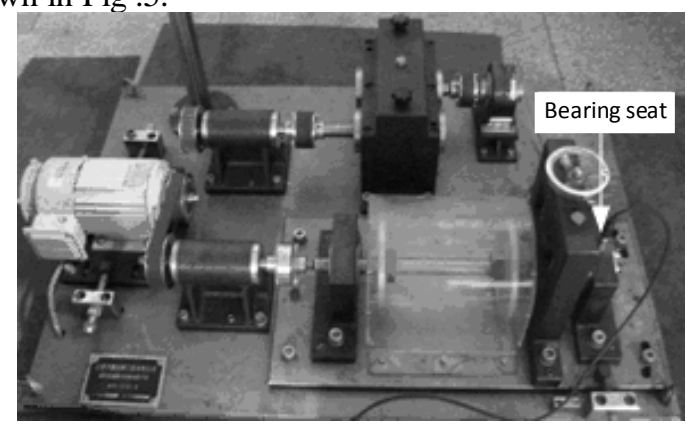

Figure 5. The experimental apparatus

This system consists of motor, belt, coupling, bearings, shaft, gear box etc. the concrete strategy was shown as following:

(1) Layout the corresponding sensors on the reasonable positions to monitor the various mechanical parts (such as gear, shaft, bearing, etc.). The types of sensor can be selected according to the specific circumstances of the object to be monitoring.

(2) Obtain the experimental data by the corresponding sensor for the monitored objects.

(3) According to the types of various original data, choose different modeling methods.

(4) Obtain the p-boxes in healthy state. 
(5) Replace the normal healthy mechanical with the fault one. In running condition, obtain the corresponding experimental data.

(6) Obtain the p-boxes in fault state.

(7) Compare of the two kinds of state (health and fault) probability box. Establish the specification of the p-box health index by some qualitative indicators.

(8) Obtain the p-boxes of all objects to be monitoring by many experiments and establish the corresponding database.

(9) Establish the mechanical parts' p-box dynamic detection index according to standard mechanical equipment state detection.

\section{CONCLUSIONS}

A new probability boxes theory research Strategy for the mechanical equipment healthy state monitoring was proposed in this paper. The probability box theory was introduced and the basic concepts and the relationship of probability box and Dampster Shafer structure were contracted to illustrate the p-box theory's capability of dealing with data uncertainty.

The relationship of probability box's compactness and data uncertainty was verified by a uncertainty Variables which obeyed by the exponential distribution. The smaller the fluctuation ranges become, the more compact the p-box express. With the parameters' fluctuation range increase, the compactness of p-box become worse, which means the uncertainties contained by p-box are increasing.

The probability box research strategy was proposed in details with a simulation rotating machinery fault test bed. The strategy may be a promising healthy state monitoring method for mechanical equipment.

\section{ACKNOWLEDGMENT}

This project is supported by National Natural Science Foundation of China (Grant No.51365020, 51369012), Yunnan Natural Science Foundation (Grant No.2013FZ020). The corresponding author of this paper is Ding Jiaman.

\section{REFERENCES}

[1] Kaufmann. Introduction to Fuzzy Arithmetic: Theory and Applications[M]. New York: Van Nostrand Reinhold, 1985.

[2] Shafer. The Combination of Evidence[J]. International Journal of Intelligent Systems, 1986, 1: 155-179.

[3] Hailperin. Boole's Logic and Probability[M]. North-Holland, Amsterdam, 1986.
[4] Frank, Nelsen and Schweizer. Best-possible bounds for the distribution of a sum - a problem of Kolmogorov[J]. Probability Theory and Related Fields, 1987, 74: 199-211.

[5] Savic. Neural generation of uncertainty reliability functions bounded by belief and plausibility frontiers[C] // In: European Conference on Safety and Reliability, 2005, 1757-1762.

[6] Nguyen, Walker. A First Course in Fuzzy Logic[M]. Boca Raton, Florida: CRC Press, 2006.

[7] Scott Ferson, Vladik Kreinovich, Lev Ginzburg, etal. Constructing Probability Boxes and Dempster-Shafer Structures [R]. California : Sandia National Laboratories, 2003.

[8] Berleant, Bounding. The Times to Failure of 2-Components Systems. IEEE Transaction on Reliability, 2004, 53(4):542-550

[9] Fulvio Tonon. Using random set theory to propagate epistemic uncertainty through a mechanical system. Reliability Engineering and System Safety, 2004, 85:169-181

[10] [10] Kriegler, Held. Utilizing belief functions for the estimation of future climate change. International Journal of Approximate Reasoning, 2005, 39:185-209

[11] Scott Ferson, Troy Tucker. Sensitivity in risk analyses with uncertain numbers. Applied Biomathematics, Setauket, New York, 2005

[12] Ferson, Tucker. Sensitivity analysis using probability bounding. Reliability engineering and system safety, 2006, 91(10): 1435-1442

[13] Limbourg, Savic. Fault tree analysis in an early design stage using the Dempster-Shafer theory of evidence. Risk, Reliability and Societal Safety. London: Aven \& Vinnem (eds) Taylor \& Francis Group, 2007

[14] Michael Oberguggenberger, Julian King, Bernhard Schmelzer. Imprecise probability methods for sensitivity analysis in engineering. In 5th International Symposium on Imprecise Probability: Theories and Applications, Prague, Czech Republic, 2007, 6: 1130-1138

[15] Destercke, Dubois, Chojnacki, Unifying practical uncertainty representations - I: Generalized p-boxes [J], International Journal of Approximate Reasoning, 2008,49(3):649-663

[16] Matthias Troffaes,Sebastien Destercke.Probability boxes on totally preordered spaces for multivariate modelling, International Journal of Approximate Reasoning, 2011,52(6):767-791

[17] Luis G. Crespo,Sean P. Kenny,Daniel P. Giesy. Reliability analysis of polynomial systems subject to p-box uncertainties, Mechanical Systems and Signal Processing, 2012,9:111-124

[18] Siao Sun,Guangtao Fu,Slobodan Djordjević,Soon-Thiam Khu. Separating aleatory and epistemic uncertainties: Probabilistic sewer flooding evaluation using probability box[J],Journal of Hydrology, 2012,420(2):360-372

[19] Matthias C.M. Troffaes, Enrique Miranda, Sebastien Destercke. On the connection between probability boxes and possibility measures Original Research Article, Information Sciences, 2013,224 (3): 88108

[20] N. Ben Abdallah, N. Mouhous-Voyneau, T. Denoeux. Combining statistical and expert evidence using belief functions: Application to centennial sea level estimation taking into account climate change[J].International Journal of Approximate Reasoning,2014, 55(1):341-354 